\title{
Investigation of the Adhesion Promoter Distribution in Porous Ceramic Precursors
}

\author{
Volker Steier, ${ }^{1}$ Christof Koplin, ${ }^{1}$ Andreas Kailer, ${ }^{1}$ and Holger Reinecke ${ }^{2}$ \\ ${ }^{1}$ Fraunhofer Institute for Mechanics of Materials IWM, Wöhlerstraße 11, 79108 Freiburg, Germany \\ ${ }^{2}$ Department of Microsystems Engineering, Laboratory for Process Technology, University of Freiburg, \\ Georges-Koehler-Avenve 103, 79110 Freiburg, Germany \\ Correspondence should be addressed to Volker Steier, volker.steier@iwm.fraunhofer.de \\ Received 31 January 2011; Accepted 31 March 2011 \\ Academic Editors: I. Emri, T. Ohara, and V. V. Zozulya
}

Copyright () 2011 Volker Steier et al. This is an open access article distributed under the Creative Commons Attribution License, which permits unrestricted use, distribution, and reproduction in any medium, provided the original work is properly cited.

The purpose of this paper is to improve the mechanical properties of polymer infiltrated ceramics (PICs) by the enhancement of the adhesion between both components. To improve the interface adhesion, an adhesion promoter (silane) was used. The silane distribution in the precursors was studied using Raman and IR spectroscopy. Inhomogeneous silane distribution was found after applying a common surface modification method. In this paper, different silane modification methods were developed. The methods differ in the manner of the infiltration solution, the point of silane activation, and the infiltration pressure. The impacts achieved by the use of these methods were analyzed, and different adhesion mechanisms between silane molecules and the ceramic surface were established. As a result, a unique infiltration method which generates homogeneous silane distributions was developed. The interface adhesion depends among other parameters on the silane amount on the surface. The adjustment of the silane amount in the precursor was realized by the use of IR-spectroscopy.

\section{Introduction}

The design of hybrid or composite materials with adapted mechanical properties by material combination and hybrid structure has already led to successfully applied materials [1, 2]. The composite materials often overcome the drawbacks of monolithic materials. Due to their excellent mechanical, biomechanical, tribological, and high-temperature properties, ceramic materials are often used as a strengthening component in composites. Despite these excellent properties, the risk of brittle fracture in monolithic ceramics restricts their applicability. Their brittleness and high elastic modulus cause such limitations. The combination of ceramics and ductile materials leads to a more favorable failure behavior, due to different strengthening mechanisms which limit fast crack propagation. The most dominant mechanisms are crack deflection and crack bridging. The latter reduces the critical crack tip stress.

The disadvantage of brittle materials is sudden failure without plastic deformation. In combination with ductile materials, the elastic modulus of the composite decreases significantly. The elastic modulus of the composites can be modified by changing the volume fraction of the components. This allows slight deformations without failure. The combination with ductile materials like metals and polymers leads therefore to a higher damage tolerance without significant reduction of the mean strength.

Nowadays, two different methods of manufacturing composites consisting of ceramic and ductile materials are applied. The first one is carried out by embedding processed ceramic particles in a ductile matrix. This method is widely used to produce materials for biomedical or engineering light-weight applications. The second manufacturing method starts with a solid but porous ceramic precursor which is infiltrated. The resulting interpenetrating matrices have higher elastic moduli and higher strength for the same ceramic polymer ratio due to the covalent-bonded ceramic matrix of this composite [3-5]. One very successful example of this type of composites is metal matrix composites (MMCs). 
<smiles>C=C(C)C(=O)OCCC[Si](C)(C)C</smiles>

(a)<smiles>C=C(C)C(=O)OCCC[Si](O)(O)O</smiles>

(b)

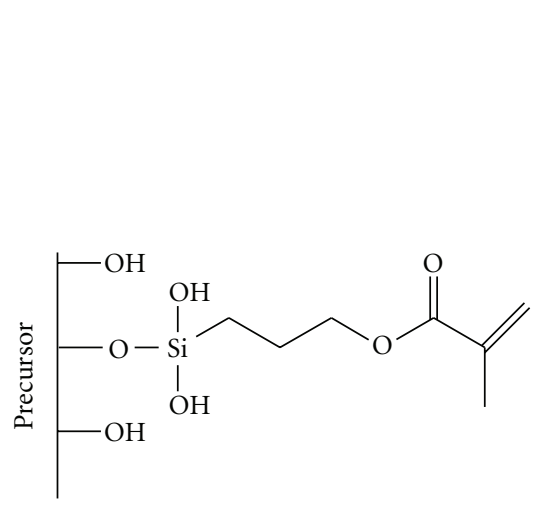

(c)

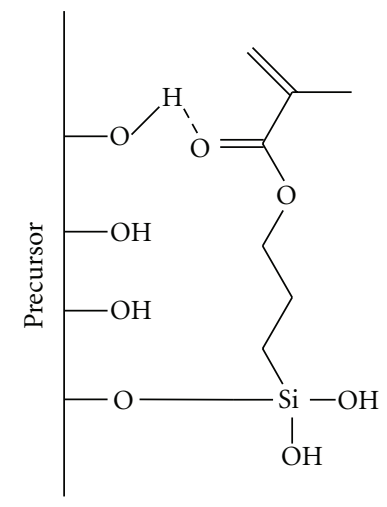

(d)

FIgURE 1: Bonding mechanisms of silane molecules.

Polymer infiltrated ceramics (PICs) are increasingly gaining the focus of today's research topics for many different applications. Besides the excellent mechanical properties and the increased fracture toughness of PICs (e.g., ceramic elastomer composites) [6], other properties like good wear resistance and biocompatibility $[4,7,8]$ make these composites attractive for tribologically demanding, bio mimetic, or medical applications $[1,9]$. Due to the possibility of changing the ceramic-to-polymer/metal ratio, the mechanical properties of the composites can be tailored for specific applications. Especially in biomedical applications, a mismatch of the elastic moduli between the artificial component and the surrounding living tissue can lead to numerous problems. Huge differences in the elastic moduli generate high interface stresses or wear particles due to interface degradation. Hence, the bio functionality of the designed material is mainly determined at the interface. Its long-term reliability is, however, governed by the surrounding living tissue (e.g., problem of stress shielding by materials with high elastic modulus).

To guarantee reliability, the components are commonly made of high-strength materials which usually possess high stiffness. An increase in toughness and failure strain increase with a reduction of stress peaks and interface loading could be observed for PICs with a reduced stiffness. With the structure of two interpenetrating matrices, the elastic modulus can be tailored to specific applications without a loss in strength.

To reach these material properties, a good interface adhesion between ceramic and polymer is needed. A strong influence of the interface bond on crack propagation and durability was established for polymer and metal ceramic composites independent of the manufacturing method [ 1 , $2,10]$. Numerous research findings have established good adhesion behavior between ceramic particles and polymers using a silane containing adhesion promoter (silane), which enable a chemical bond between the organic and inorganic components $[11,12]$. The surface modification of ceramic filler particles can be easily realized by mixing the particles in a silane solution. In contrast, the porous structure and the predominant internal surface of a ceramic precursor hinder an easy surface modification. Due to the requirement of high interface adhesion to reach high mechanical strength of PICs, a sufficient silane coating is required.

Adhesion promoter distribution in porous precursors has not been determined up to now. A current surface modification method is achieved by infiltrating the precursor with a silane solution. Inhomogeneous silane distributions are assumed by the use of this method. Hence, a measurement method to investigate the silane distribution in the precursors has to be found. The further aim of this study is to understand the bonding mechanisms of the silane molecules with the ceramic surface and to develop an infiltration method, which leads to a homogeneous silane distribution. Besides a sufficient surface modification, the amount of silane influences the interface strength. To achieve the overall goal of high interface strength, the silane amount has to be adjusted to a structure specific value.

\section{Materials and Methods}

Two different ceramic precursors were used. Silane distribution tests were carried out on zirconium oxide specimens $\left(14 \times 15 \times 40 \mathrm{~mm}^{3}\right.$, VITA Zahnfabrik, Germany) with an average pore radius of $70 \mathrm{~nm}$ and a porosity of $50 \%$. The determined results were verified using aluminum oxide precursors. Due to equivalent results, the determination of a sufficient silane concentration for the infiltration solution 
TABLE 1: Infiltration methods and specimens.

\begin{tabular}{|c|c|c|c|c|c|}
\hline $\begin{array}{l}\text { Specimen } \\
\text { number }\end{array}$ & Method & Silane status & Drying & $\begin{array}{l}\text { Drying } \\
\text { position }\end{array}$ & Silanization solution \\
\hline 1 & Partial immersion method (PI) & Activated & Yes & Normal & $\begin{array}{l}48.5 \text { vol. } \% \text { ethanol, } 48.5 \text { vol. } \% \text { water, } \\
3 \text { vol. } \% \text { silane*, } \text { pH } 3^{-}\end{array}$ \\
\hline 2 & Infiltration with inactivated silane & Inactivated & Yes & Normal & 97 vol.\% ethanol, 3 vol.\% silane* \\
\hline 3 & Infiltration with inactivated silane & Inactivated & Yes & Normal & 97 vol. $\%$ ethanol, 3 vol. $\%$ silane* \\
\hline 4 & Infiltration with inactivated silane & Inactivated & Yes & Upside down & 97 vol. $\%$ ethanol, 3 vol. $\%$ silane* \\
\hline 5 & Infiltration with inactivated silane & Inactivated & Yes & Normal & 97 vol. $\%$ ethanol, 3 vol. $\%$ silane* \\
\hline 6 & Delayed activation method (DA) & $\begin{array}{l}\text { Delayed } \\
\text { activation }\end{array}$ & No & Normal & $\begin{array}{l}\text { First infiltration: } 97 \text { vol.\% ethanol, } \\
3 \text { vol.\% silane* } \\
\text { Second infiltration: Water with a } \mathrm{pH} \text { of } 3\end{array}$ \\
\hline 7 & High flow infiltration (HFI) & Activated & Yes & Normal & $\begin{array}{l}48.5 \text { vol. } \% \text { ethanol, } 48.5 \text { vol. } \% \text { water, } \\
3 \text { vol. } \% \text { silane*, pH } 3\end{array}$ \\
\hline
\end{tabular}

*Evonik Industries AG, Germany.

was carried out on aluminum oxide precursors $(10 \times 12$ $\times 16 \mathrm{~mm}^{3}$, VITA Zahnfabrik, Germany). The average pore radius of the aluminum oxide specimens was $113 \mathrm{~nm}$ and the porosity of the specimens was $24 \%$.

After activation of the silane molecules, they form chemical bonds to the ceramic surface. In Figure 1 the reaction steps are shown. The first illustration shows the inactivated silane molecule (Figure 1(a)). The activation reaction occurs by hydrolysis. Via protonation of hydrogen atoms on the methyl groups, methanol groups are separated from the silane molecules. After separation, reactive hydroxyl groups are formed at the unsaturated silicon (Figure 1(b)) [13-15]. These silanol groups are reactive and form chemical bonds via condensation reactions with the ceramic surface or with each other (appearance of siloxane films). There are two types of bonding with the precursor.

There are two types of bonding with the precursor, where the silane molecules can align perpendicular or parallel to the surface (Figures 1(c) and 1(d)). The type of bonding can be detected by IR spectroscopy and it mainly depends on the silane concentration of the infiltration solution [11, 12]. Hence, the infrared analysis was used to adjust the silane concentration of the infiltration solution. As shown in Figure 1, the silane molecules consist of a methacryl group. This molecular structure contains $\mathrm{C}=\mathrm{C}$ and $\mathrm{C}=\mathrm{O}$ bonds. These bonds can also be detected via characteristic peaks in the Raman spectrum. In Figure 2, the Raman spectrum of silane molecules on $\mathrm{ZrO}_{2}$ in the range of $1200 \mathrm{~cm}^{-1}-1900 \mathrm{~cm}^{-1}$ is shown. To characterize the silane distribution semiquantitatively, the area under the $\mathrm{C}=\mathrm{C}$ peak for every single measurement was computed. This area can be correlated to the amount of silane molecules on the surface (Figure 2). The silane distribution was determined by line scans on cut surfaces with aligned measurement points (distance $100 \mu \mathrm{m}$; Figure 3(c)).

In a Raman spectrum, the methacryl group within the silane molecules can be detected. Stretching vibrations of the $\mathrm{C}=\mathrm{C}$ bond at $1638 \mathrm{~cm}^{-1}$ were used for the determination of the silane distribution in the precursor. To determine the

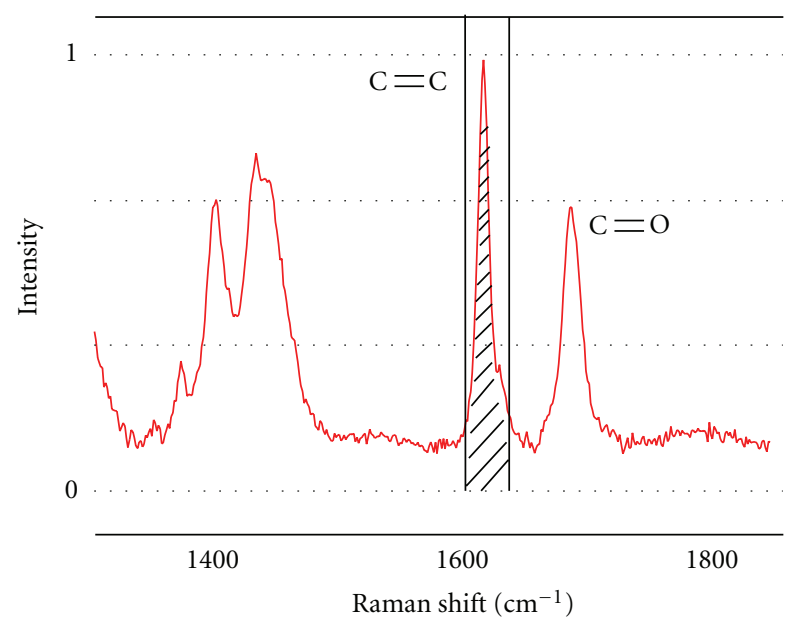

Figure 2: Raman spectrum of silane on $\mathrm{ZrO}_{2}$ with a schematic illustration of the calculation method.

best possible silane distribution, three different infiltration methods were investigated. The infiltrated specimens and the silanization parameters are shown in Table 1. Independent of the infiltration method, all specimens (except specimen no. 2) were dried in an oven at $60^{\circ} \mathrm{C}$ under a pressure of $200 \mathrm{~Pa}$ for $10 \mathrm{~h}$ after the infiltration. Low pressure ensures the removal of all volatile components (water and ethanol are in the vapor phase at this pressure).

To investigate the silane distribution in zirconium oxide precursors, a partial immersion infiltration method was used (PI method). The precursors were immersed in the modification solution; the complete infiltration occurs due to capillary forces. The infiltrations were realized in a pressure chamber at a pressure of $20 \mathrm{kPa}$. After a two-hour holding time at this pressure, the lower portion of the cube-shaped specimens were immersed in the infiltration solution (Figure 3(a)). This ensured removing entrapped air via the top plane. Entrapment of air could occur if 

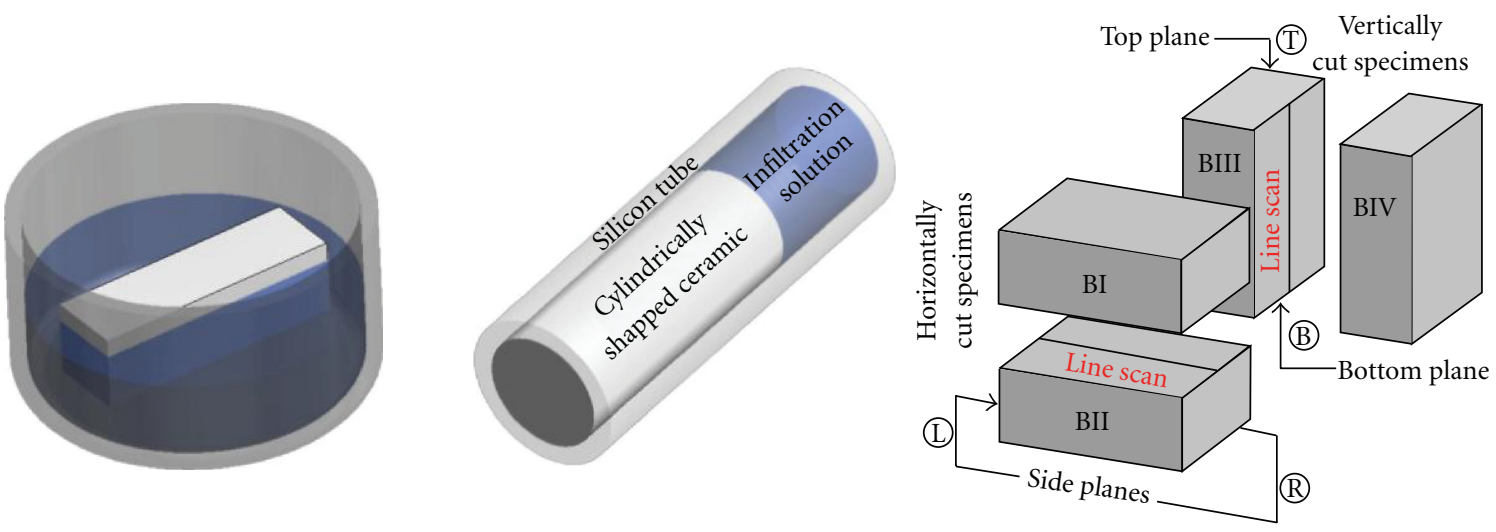

FIgURe 3: Infiltration devices and specimen preparation before scanning. (a) Partially immersed specimen (PI), (b) high flow infiltration device (HFI), and (c) specimen separation before spectroscopic analyses.

the specimens were completely submerged in the solution. The specimens were partially immersed for 1 hour, then completely submerged in the solution, where the pressure was brought to an atmospheric pressure.

The method, we denoted "DA method", differs to some extent from the PI method. For this method two separate infiltration steps are realized. The immersion procedure is always the same, but the infiltration solution is different for each step. In the first infiltration step water is not used. Therefore, the hydrolysis of the $-\mathrm{CH}_{3}$ molecules does not start. By doing this, no chemical bonds between the silan molecules and the surface can be formed to keep the silane molecules mobile. The precursors were then dried in an oven. For the second infiltration step, a mixture of water with ascetic acid at a $\mathrm{pH}$ of 3 was used to start the hydrolysis in the pretreated ceramics. After the infiltration, the specimens were stored in this solution for $12 \mathrm{~h}$ to ensure a high silane activation rate.

The "HFI" method was used to investigate the effect of an increased silane circulation inside the ceramic. To ensure an adequate sealing, the first HFI tests were performed with cylindrically shaped precursors. After the precursor was fitted and fixed in a reinforced silicon tube (Figure 3(b)), the activated silane solution was pressed with a pressure of 1.2 MPa through the ceramic (infiltration time: $12 \mathrm{~h}$ ).

To study the silane distribution inside the ceramic precursors, the specimens 1-6 (see Table 1) were cut into four pieces. The front section was cut horizontally and the back section vertically (Figure 3(c)). Two horizontal measurements and two vertical measurements were carried out. The single precursor blocks were labeled, to distinguish the horizontally (BI, BII) and vertically (BIII, BVI) cut specimens. The specimen position during the infiltration was also labeled, by the initial letter of the positions (bottom, top, left, right).

For specimen no. 7 which was infiltrated with the HFI method, only one lengthwise cut was necessary. This cut allows measuring the silane distribution along and across the flow direction. As a surface preparation for spectroscopic measurements, the specimens were polished with an abrasive paper, cleaned in an ultrasonic bath, and dried again in an oven (conditions: see above).

\section{Results}

In the pretests, a silane deposition on dense sintered ceramic surfaces indicating various crosslinking behaviors was observed. After drying at $60^{\circ} \mathrm{C}$, the layer could easily be removed from the surface (by simple scratching), in contrast to the specimens dried at $100^{\circ} \mathrm{C}$, where traces of the silane remained on the surface. There are two possibilities for a chemical bond between silane molecules. (1) A linkage via Si$\mathrm{O}-\mathrm{Si}$ bonds and a (2) crosslinking polymerization of the $\mathrm{C}=\mathrm{C}$ bonds to C-C chains. After drying at a temperature of $100^{\circ} \mathrm{C}$ the $\mathrm{C}=\mathrm{C}$ bond intensity significantly decreases (Figure 4 ), indicating that polymerization of the silane occurs.

Accordingly, to avoid an influence of the drying process on the analysis, the precursors were dried at a temperature of $60^{\circ} \mathrm{C}$ for the subsequent analysis.

The intensities of the $\mathrm{C}=\mathrm{C}$ modes are clearly varying within the ceramic samples (Figure 5). The local measured intensity over the specimens surface (Figure 3 ) was scanned, to determine the amount of silane and its bonding mechanisms. To compare the measurements of different specimens with each other, the computed data were normalized by dividing by the highest intensity.

For the PI method (specimen 1, Figure 5(a)) high silane accumulations were found in the vicinity of the right and left edges $(R, L)$ of the horizontally cut blocks (BI, BII), that is, the original side planes. The vertically cut blocks (BIII, BVI) show high silane intensities next to the bottom plane (B), in contrast to the low silane intensity close to the top plane $(\mathrm{T})$. There is a decrease in the amount of silane in the direction of the specimen center.

To describe these results of the delayed activation method (DA method), all intermediate steps were clarified separately. The silane distribution measured immediately after the infiltration with inactivated silane molecules is homogeneous (specimen 2, Figure 5(b)) with regard to the microstructure of the specimens. The blocks were not dried before scanning. 


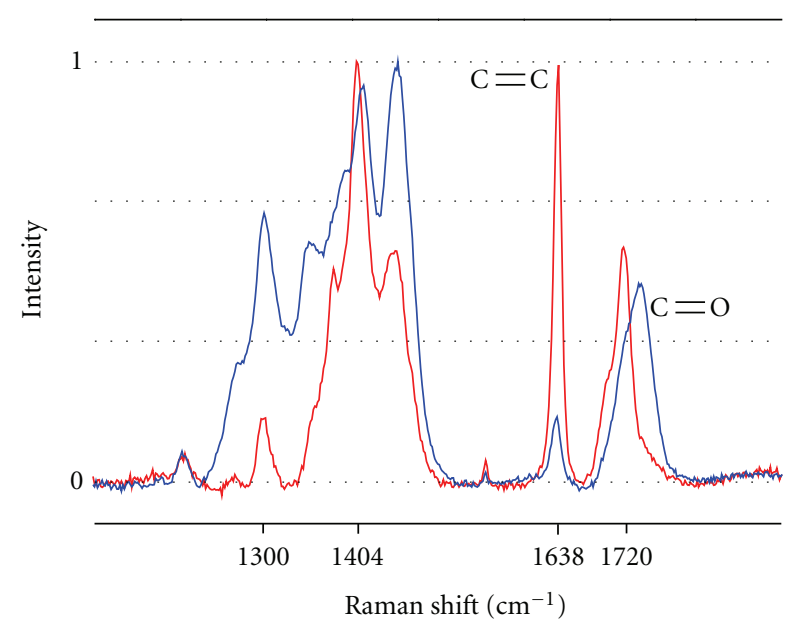

Figure 4: Raman spectrum of silane. (a) Differences of the Raman spectrum due to different drying conditions (200 Pa); blue graph: $100^{\circ} \mathrm{C}$, red graph: $60^{\circ} \mathrm{C}$.

After the drying process (specimen 3, Figure 5(c)) high concentrations of silane at both side planes $(\mathrm{L}, \mathrm{R})$ and at the top plane $(\mathrm{T})$ were measured. In contrast to specimen 1 , the highest silane concentration was measured at the top plane.

The influence of the drying position was examined, for which reason only the results of the vertical cut specimens (BIII, BVI, Figure 5(d)) are shown. If the specimens were dried in the same position as the infiltration was realized (top plane on top, specimen 4), the highest amount of silane was found on the top plane. For the specimens that were dried upside down (top plane downwards, specimen 5), the highest amount of silane was found on the bottom plane. Hence, an influence of the drying step was proved.

After all the described intermediate steps, the DA method (specimen 6, Figure 5(e)) yields the highest silane concentrations in the center of the specimens independent of the cutting direction.

For specimen 7 (HFI method), a nearly homogenous silane distribution was found (Figure 5(f)). In the lengthwise scanning direction, the highest silane intensity was found at the emersion side. The perpendicular scan revealed a slightly higher silane amount. Due to the small variation of the local silane concentration, the silane distribution can be regarded as homogeneous.

To verify the results measured with Raman spectroscopy in zirconium oxide precursors, the zirconium oxide specimens were also scanned with an IR-spectrometer. Furthermore, a different ceramic material (aluminum oxide) was used to prove the influence of the precursor material on the silane distribution in the precursor. As a result, similar silane distributions were found using aluminum oxide precursor and the described infiltration methods.

Beside a homogeneous silane distribution, the amount of silane on the surface influences the interface strength between ceramic and polymer. Hence, the silane concentration of the infiltration solution has to be adjusted. To determine the desired silane layer thickness, silane concentration tests were realized with aluminum oxide precursors.
Since the HFI method generates homogeneous silane distributions, this method was chosen for the adjustment of the silane concentration. As described above (Figure 1), the mean bonding characteristics of silane molecules on the surface can be distinguished by IR spectroscopy. In Figure 6(a), the $\mathrm{C}=\mathrm{O}$ peak shift between $1700 \mathrm{~cm}^{-1}$ and $1730 \mathrm{~cm}^{-1}$ is shown. The peak position is an indicator for the main molecule orientation and also for the silane amount on the surface.

A $C=O$ peak shift to higher wave numbers is an indicator of perpendicular surface bonding. Parallel surface bonding mainly occurs at low silane concentrations whereas high silane concentrations correspond to perpendicular molecule orientation. Hence, the silane amount on the surface can be determined by the shift position. The intensity of the peak at $1730 \mathrm{~cm}^{-1}$ in Figure 6(a) is much higher than the intensity at $1700 \mathrm{~cm}^{-1}$. The difference in intensity shown in Figure 6(a) supports this fact.

The spectra shown in Figure 6(b) were measured in a line scan on an aluminum oxide specimen. The uppermost and the lowest spectra represent the specimen edges. The distance between the measurement points was $0.5 \mathrm{~mm}$. With regards to the above-described silane molecule orientation, a $\mathrm{C}=\mathrm{O}$ peak at $1710 \mathrm{~cm}^{-1}$ and a smaller peak at $1720 \mathrm{~cm}^{-1}$ are indicators of a thin silane layer with perpendicular and parallel orientated silane molecules. With the exception of the last measurement at the specimens edge, the peak level of all $\mathrm{C}=\mathrm{O}$ peaks are nearly identical. Silane concentrations between $3 \%$ and $0.2 \%$ were used to receive a sufficient but thin silane layer on the ceramic surface. The shown spectra in Figure 6(b) represent such a silane layer. The used silane concentration was 0.36 vol. $\%$.

To summarize the results, the silane distribution in ceramic precursors was significantly improved. Initially, inhomogeneous distribution of silane was observed in the precursors after the infiltration with a common immersion method. To raise the silane amount in the precursor center, an infiltration with delayed activation was investigated. Although a homogeneous silane concentration could not be reached with this method, other important influencing factors were found. A homogeneous silane distribution was achieved using the HFI method. The silane distribution measurements were realized with a Raman spectrometer. To enable good adhesion properties, an IR spectrometer was used to adjust the silane concentration in the infiltration solution.

\section{Discussion}

To achieve homogeneous material behavior and excellent mechanical properties in PICs, a sufficient interface adhesion should be ensured. Hence, a complete and homogeneous modification of the whole internal surface with adhesion promoter is needed. The aim of this study was to optimize the silane distribution in porous ceramic precursors to reach high interface strength between ceramic and polymer after subsequent monomer infiltration and polymerization. Therefore, the mechanism of silane bonding to the ceramic surface had to be studied. The challenging problem for this 


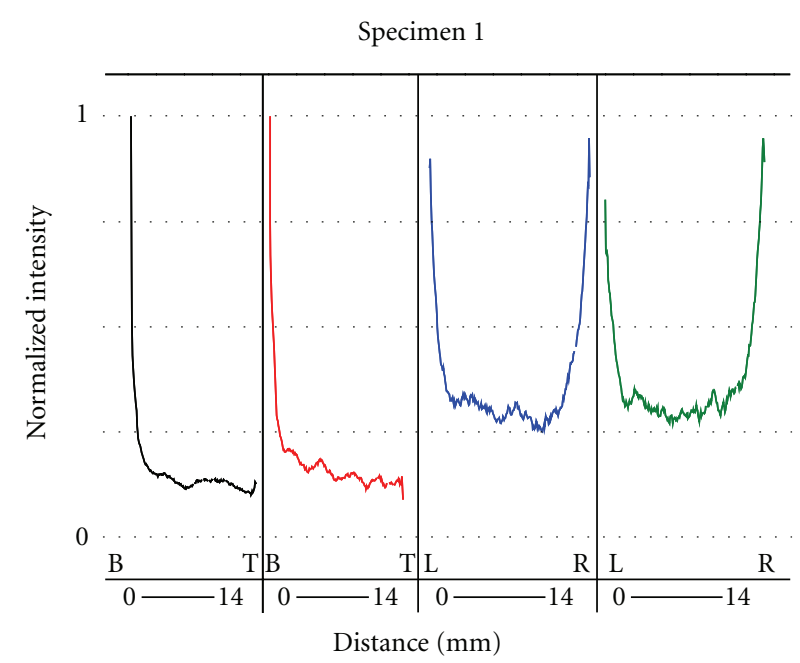

(a)

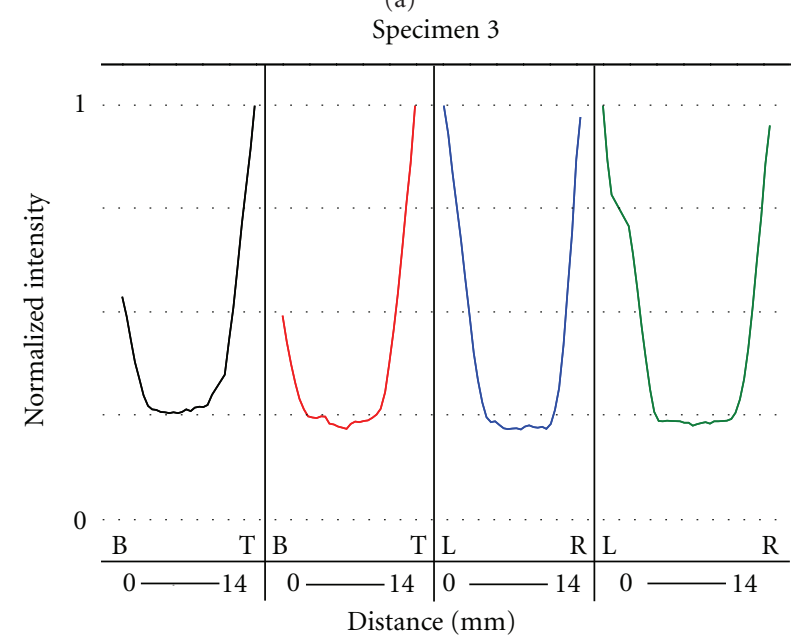

(c)

Specimen 6

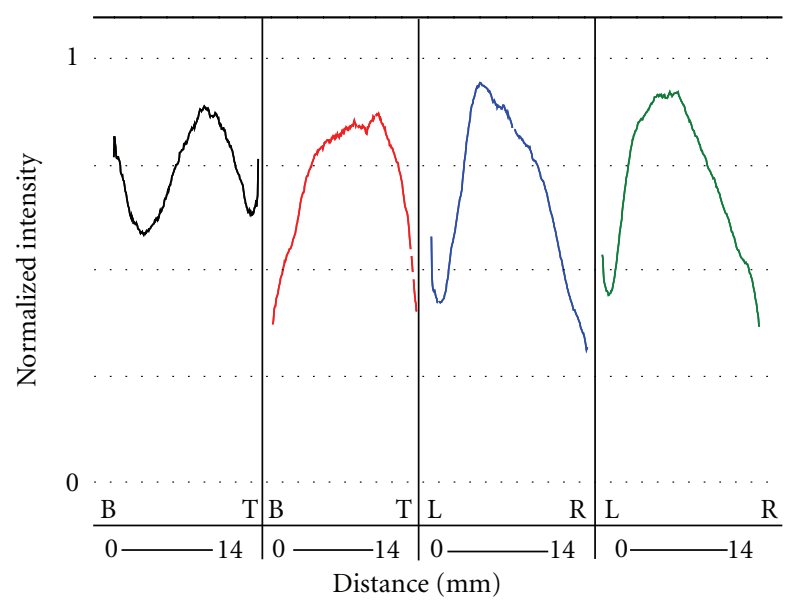

(e)

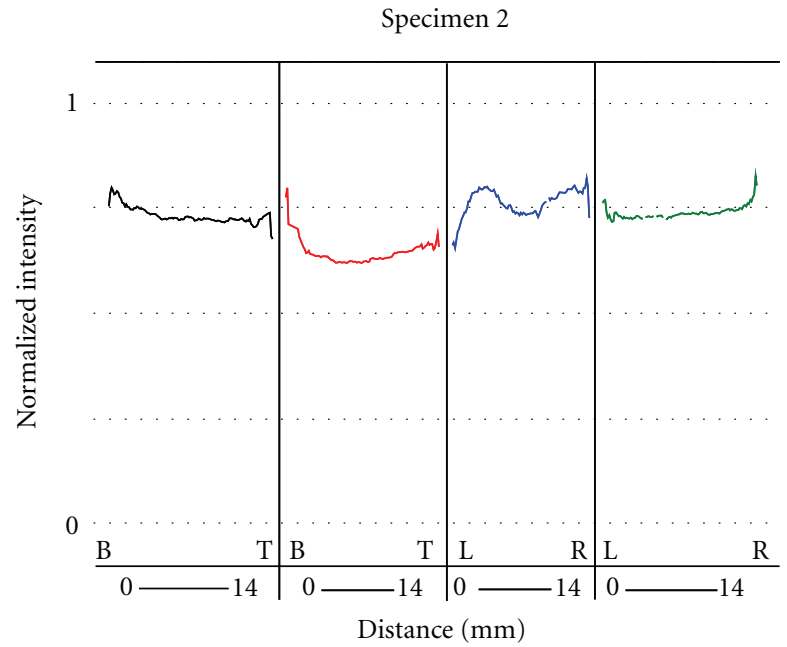

(b)

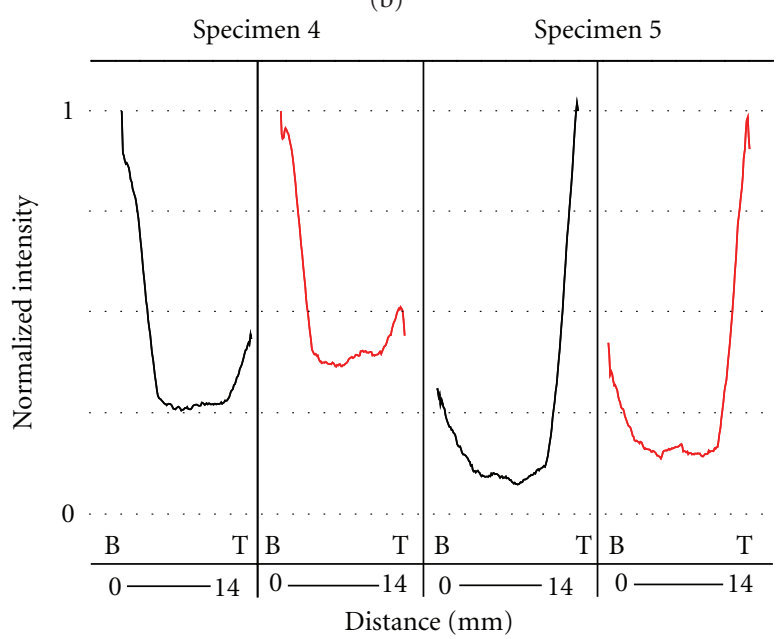

(d)

Specimen 7

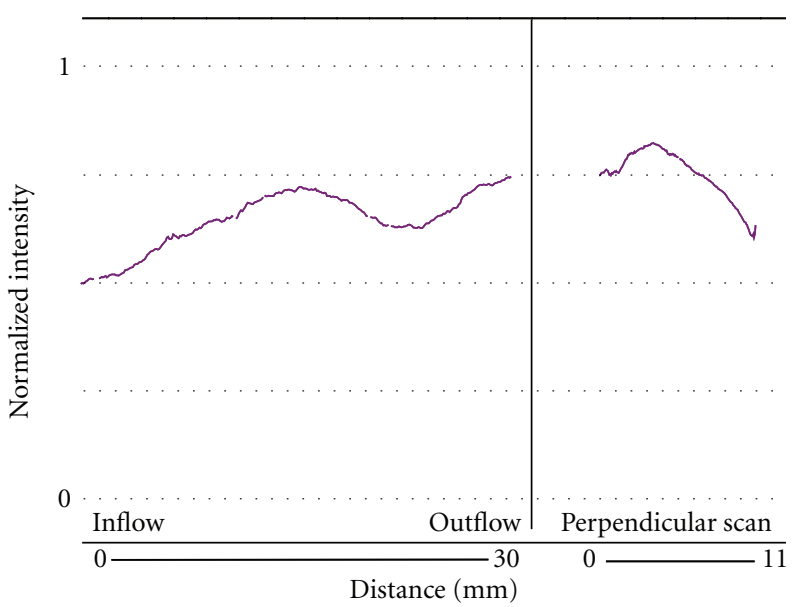

(f)

FIGURE 5: Silane distribution in zirconium oxide specimens. (a) PI method: first graph vertical cut (BIII), second graph vertical cut (BVI), third graph horizontal cut (BI), and fourth graph horizontal cut (BII). (b) Scan after infiltration with inactivated silane molecules without drying: first graph BIII, second graph BVI, third graph BI, and fourth graph BII. (c) Scan after infiltration with inactivated silane molecules and drying: first graph BIII, second graph BVI, third graph BI, and fourth graph BII. (d) Scan after infiltration with inactivated silane solution and drying: first graph BIII dried upside down, second graph BVI dried upside down, third graph BIII normal drying, and fourth graph BIV normal drying. (e) DA method: Scan after second infiltration with water: first graph BIII, second graph BVI, third graph BI, and fourth graph BII. (f) HFI method: first graph scanned in flow directionand second graph scanned perpendicular to flow direction. 


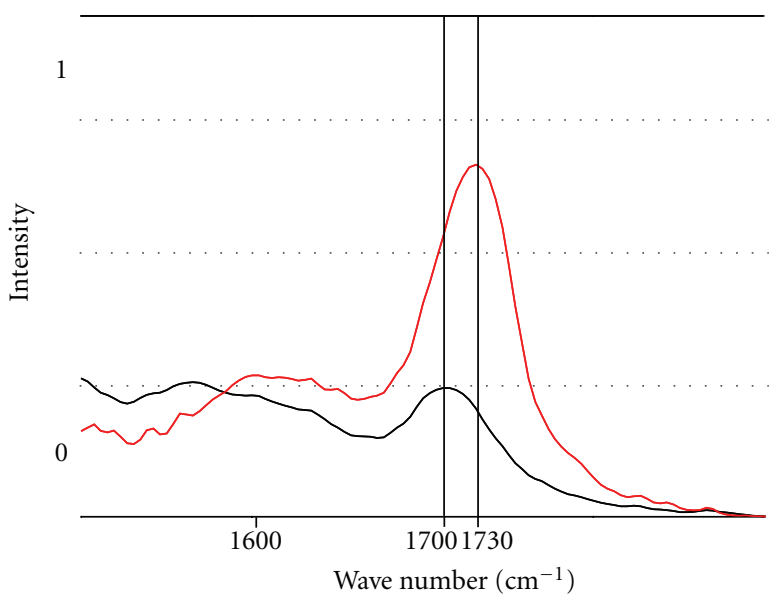

(a)

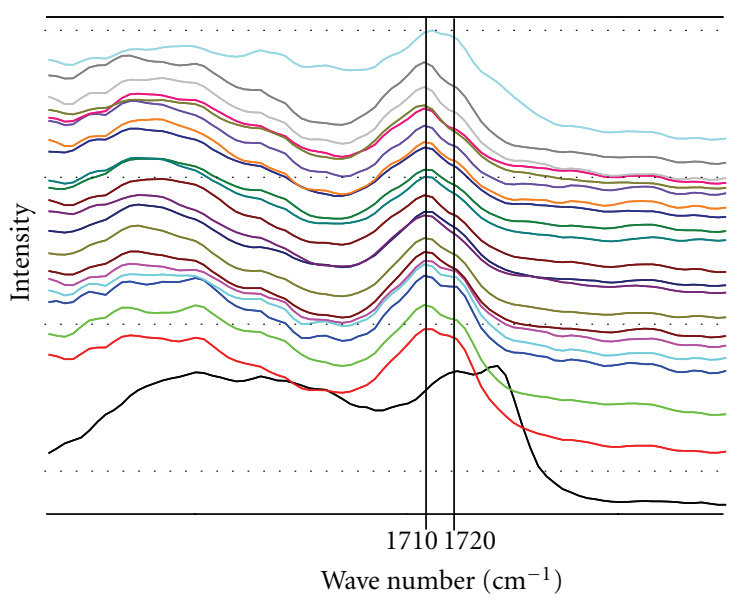

(b)

FIgURE 6: IR spectra. (a) IR spectrum of silane on $\mathrm{Al}_{2} \mathrm{O}_{3}$. Shift of $\mathrm{C}=\mathrm{O}$ peak; red graph: high silan concentration, black graph: low silane concentration. (b) 20 IR spectra in the range of 1530 to $1850 \mathrm{~cm}^{-1}$. Each spectrum represents a measurement point on a line scan, measured analogous to the in Figure 3(c) described system (Topmost spectrum = top plane; lowermost spectrum = bottom plane; used silane concentration $0.36 \%)$.

process is the difficulty of accessing the surface inside the ceramic matrix. The various influencing effects that were found for the described methods will be discussed separately. The PI method is a straightforward infiltration approach with the result that infiltration, silan, activation, and drying are interacting processes that have to be separately studied. This was intended with the design of the DA method. All the results led to the final method (HFI) that will be discussed in the end.

The results of the first applied PI infiltration method show high silane concentrations only in limited parts of the specimens. Unlike the horizontally cut specimens, the vertically cut specimens show a high silane concentration only close to the bottom plane (B; specimen 1, Figure 5(a)). Due to the partial immersion, the silane concentration decreases towards the center and the top plane $(\mathrm{T})$. An immediate chemical bonding to the ceramic surface during the infiltration process leads to the high silane concentrations near the edges. The small pore size, the branched microstructure, and the rough surface hinder a fast flow of the silane molecules through the ceramic precursor. The occurrence of the highest silane concentration at the bottom plane $(\mathrm{B})$ is an indicator of an immediate surface bonding during the infiltration procedure. If no permanent surface adhesion exists, the highest silane concentration would occur at the top plane (T; refer to specimen 3, Figure 5(c)). The silane concentration in the infiltration solution decreases with the infiltration distance due to a direct bonding to the surface.

A size filtering effect can be excluded due to the molecule size of about $1 \mathrm{~nm}$ and a mean pore radius of $70 \mathrm{~nm}$. Previous investigations detect a remaining of $70 \%$ activated $\mathrm{Si}-\mathrm{OH}$ groups after the applied activation time [16]. This means that no large SiOSi chains were formed, and large silane agglomerates in the dimension of the average pore radius can be excluded.
Due to the direct bonding of the silane molecules, an infiltration method with delayed activation was tested before the DA method. To describe all the occurring adhesion and redistribution processes happening during the intermediate steps, each influencing factor is described in detail.

The silane distribution shown in Figure 5(b) (specimen 2) was measured immediately after the infiltration without any drying. In this measurement on soaked specimens, the silane molecules were equally distributed. The differences are not significant and occur due to the microstructure of the precursor. However, the silane molecules are not activated and only physical surface adhesion is possible.

The results illustrated in Figure 5(c) (specimen 3) show the silane distribution after the trailed drying process. The highest silane concentrations occur next to the side $(\mathrm{L}, \mathrm{R})$ and top planes $(\mathrm{T})$. This behavior can be explained by redistribution during the drying step. It is assumed that the evaporation of the ethanol mainly occurs on the side and the top planes, where the only physically bonded silane molecules were transported towards side and top planes (L, $\mathrm{R}, \mathrm{T}$ ). On the bottom plane, no significant evaporation was possible, due to the supporting surface in the oven.

To study the influence of drying in detail, two further infiltrations were realized. In this case, the position in the oven during the drying process was changed. Figure 5(d) shows four graphs from two vertically cut specimens (specimen 4 and 5). The first two graphs illustrate the silane distribution of the "upside down" dried specimen number 4. The third and the fourth graph show the silane distribution of specimen 5, which was dried in a normal position. Here it can be shown that the highest silane concentration occurs at the upper facing surface during the drying process regardless of its position during infiltration. The location of the highest silane concentration is therefore clearly dependent on the position during drying for the infiltration with inactivated 
silane molecules. To summarize the results of an infiltration with inactivated silane molecules, any performed drying step after the infiltration leads to a redistribution of the silane molecules and therefore to high silane concentrations at the specimens edges.

Therefore the immersion method was modified by realizing a second infiltration step with water at a $\mathrm{pH}$ of 3 to activate the silane molecules (DA method). By this infiltration, the only adsorbed silane molecules were carried towards the center of the specimens. At the same time the silane molecules were hydrolyzed and they were able to form chemical bonds with the precursor. The highest silane concentration occurs in the center of specimen 6 (Figure 5(e)). The existence of regions with no or very little silane amounts near the side planes after the drying step is a further argument for direct silane adhesion to the ceramic surface after activation. Both described immersion methods, PI and DA, do not show a homogeneous silane distribution.

The aim of the HFI method was to raise the infiltration flow inside the precursor, which results in a nearly homogeneous silane distribution (specimen 7, Figure 5(f)). Only a slight increase in the silane concentration in flow direction was measured. This finding is significantly different from the results of the PI and the DA method (specimen 1 and 6), where silane molecules immediately formed chemical bonds with the ceramic surface after the activation. If this behavior is assumed, the silane concentration should decrease in flow direction. The combination of the pressure-assisted flow and the interaction of silane molecules with water is responsible for the improved silane distribution. In their nonactivated state, the silane molecules are strongly hydrophobic. After the hydrolysis, they transform to hydrophilic molecules. Following the adhesion on the surface and the formation of siloxane films, they become hydrophobic again [17]. The interaction between the silane and the surface occurs in the hydrophilic state. This behavior hinders an immediate surface bonding due to the waterless infiltration solution. The applied flow through the precursor flushes a considerable amount of silane molecules out of the precursor, which do not have sufficient surface contact. Drying by airflow, which started directly after the liquid infiltration flow, caused the evaporation of infiltration solution within the porous precursor microstructure, which presumably left behind bonded silane molecules. According to the silane distribution shown in Figure 5(f), it is assumed that the silane accumulation follows the airflow direction. The perpendicular scan also shows slight differences in the silane distribution. Due to the porosity distribution resulting from the ceramic compaction process [18], the most porous section is the center of the specimens. The change in the microstructure and the changed pore volume rate can be responsible for the slightly measured differences.

The IR measurements support the results of the Raman analysis. The silane distribution generated with the HFI method is nearly homogeneous. The aim of this test series was to find a silane concentration for the infiltration solution, which leads to a complete surface coverage and therefore to good adhesion. A decrease of adhesion strength in the case of too much silane on the surface was observed by other researchers [1], for which reason thick silane layers on the surface should be avoided. As described above, the signal maximum of the $\mathrm{C}=\mathrm{O}$ peak can shift between $1700 \mathrm{~cm}^{-1}$ and $1730 \mathrm{~cm}^{-1}$ in the FTIR spectrum (Figure 6(a)). The shift can be related to the amount of silane bonded to the surface $[12,19,20]$. If the carbonyl group of the silane molecule has no interactions neither with the surface (Figure 1(c)) nor with other silane molecules (hydrogen bonds are possible), the carbonyl peak shifts to higher wave numbers. The peak center at $1730 \mathrm{~cm}^{-1}$ is therefore an indicator of a huge silane amount. If the carbonyl group is hydrogen bonded (Figure 1(d)), and therefore orientated parallel to the surface, the peak will occur at $1700 \mathrm{~cm}^{-1}$. Peaks at $1700 \mathrm{~cm}^{-1}$ represent a low silane amount. The signal intensity correlated to the peak position of the shown $\mathrm{C}=\mathrm{O}$ bond in Figure $6(\mathrm{a})$, verifies this behavior. With a silane concentration of $0.36 \mathrm{vol} . \%$, the highest $\mathrm{C}=\mathrm{O}$ peak occurred at $1710 \mathrm{~cm}^{-1}$. A second, smaller $\mathrm{C}=\mathrm{O}$ peak was measured at $1720 \mathrm{~cm}^{-1}$, which represents the beginning of silane accumulation. The present silane layer can therefore be assumed as homogeneous and thin. Due to the performed line scan spectra analysis on aluminum oxide specimens, the IR measurements confirm the results of Raman measurements with zirconium oxide specimens. Hence, the bonding mechanisms determined on zirconium oxide and the used silane molecules can be transferred to other ceramic materials.

\section{Conclusions}

For the investigated PICs, good mechanical properties were expected if a sufficient and homogeneous silane distribution can be generated. To improve the silane distribution and the mechanical properties of PICs, different infiltration methods were studied. The detection of the silane distribution in porous ceramic precursors was realized using Raman and IR spectroscopy. As expected, the common used immersion method leads to inhomogeneous silane accumulations in the precursor. The use of a delayed activation method gave important indications on the bonding mechanisms of silane with the ceramic surface in their inactivated and their activated state. Furthermore, the silane distribution in the specimens showed a dependency on the drying conditions and the point of silane activation. Due to the redistribution of inactivated silane molecules during drying, a homogeneous silane distribution could not be reached with delayed activation, though the silane distribution was homogenous after infiltration with inactivated silane molecules. The use of a pressure-assisted infiltration method which raises the infiltration flow inside the precursor leads to a homogeneous silane distribution. With the use of this method a homogeneous silane distribution and therefore excellent mechanical properties can be expected.

Beside a homogeneous silane distribution, the silane amount on the surface is important for a good interface adhesion. With IR spectroscopy, a semiquantitative determination of the silane amount on the surface was realized. In the case of using a silane concentration of $0.36 \mathrm{vol} . \%$ and the aluminum oxide specimens, the $\mathrm{C}=\mathrm{O}$ peak occurs at 
$1710 \mathrm{~cm}^{-1}$, which represents an adequate surface coverage without the formation of bulk like silane layers. The subsequent investigations will focus on the influences of the interface adhesion on the mechanical properties of PICs.

\section{Acknowledgments}

The authors acknowledge the financial support of the German Research Foundation (DFG) under Grant KO 3872/1-1. Thanks are also due to the coworkers at the Fraunhofer Institute for Mechanics of Materials IWM and the Department of Microsystems Engineering in Freiburg for their help and support.

\section{References}

[1] K. S. Wilson, K. Zhang, and J. M. Antonucci, "Systematic variation of interfacial phase reactivity in dental nanocomposites," Biomaterials, vol. 26, no. 25, pp. 5095-5103, 2005.

[2] A. Neubrand, T.-J. Chung, and J. Rödel, "Experimental and theoretical investigation of R-curve behaviour in $\mathrm{Al} / \mathrm{Al} 2 \mathrm{O} 3$ functionally graded materials," Materials Science Forum, vol. 423-425, pp. 269-274, 2003.

[3] M. Peroglio, L. Gremillard, J. Chevalier, L. Chazeau, C. Gauthier, and T. Hamaide, "Toughening of bio-ceramics scaffolds by polymer coating," Journal of the European Ceramic Society, vol. 27, no. 7, pp. 2679-2685, 2007.

[4] R. H. Plovnick and D. J. Pysher, "Strengthening low-density silicon nitride beads via an inorganic polymer route," Materials Research Bulletin, vol. 35, no. 9, pp. 1453-1461, 2000.

[5] M. T. Tilbrook, R. J. Moon, and M. Hoffman, "On the mechanical properties of alumina-epoxy composites with an interpenetrating network structure," Materials Science and Engineering, vol. 393, no. 1-2, pp. 170-178, 2005.

[6] A. Boczkowska, K. Konopka, and K. J. Kurzydowski, "Effect of elastomer structure on ceramic-elastomer composite properties," Journal of Materials Processing Technology, vol. 175, no. 1-3, pp. 40-44, 2006.

[7] J. M. Gómez de Salazar, M. I. Barrena, G. Morales, L. Matesanz, and N. Merino, "Compression strength and wear resistance of ceramic foams-polymer composites," Materials Letters, vol. 60, no. 13-14, pp. 1687-1692, 2006.

[8] K. Konopka, A. Boczkowska, K. Batorski, M. Szafran, and K. J. Kurzydyowski, "Microstructure and properties of novel ceramic-polymer composites," Materials Letters, vol. 58, no. 30, pp. 3857-3862, 2004.

[9] R. Prehn, F. Haupert, and K. Friedrich, "Sliding wear performance of polymer composites under abrasive and water lubricated conditions for pump applications," Wear, vol. 259, no. 1-6, pp. 693-696, 2005.

[10] N. M. Mohsen and R. G. Craig, "Effect of silanation of fillers on their dispersability by monomer systems," Journal of Oral Rehabilitation, vol. 22, no. 3, pp. 183-189, 1995.

[11] A. Karmaker, A. Prasad, and N. K. Sarkar, "Characterization of adsorbed silane on fillers used in dental composite restoratives and its effect on composite properties," Journal of Materials Science: Materials in Medicine, vol. 18, no. 6, pp. 1157-1162, 2007.

[12] J. D. Miller and H. Ishida, "Quantitative monomolecular coverage of inorganic particulates by methacryl-functional silanes," Surface Science, vol. 148, no. 2-3, pp. 601-622, 1984.

[13] H. Ishida and J. L. Koenig, "Reinforcement mechanism of fiber-glass reinforced plastics under wet conditions: a review,"
Polymer Engineering and Science, vol. 18, no. 2, pp. 128-145, 1978.

[14] H. Ishida and J. L. Koenig, "Vibrational assignments of organosilanetriols. II. crystalline phenylsilanetriol and phenylsilanetriol- $\mathrm{d}_{3}$," Applied Spectroscopy, vol. 32, no. 5, pp. 469-479, 1978.

[15] J. P. Matinlinna, L. V. J. Lassila, I. Kangasniemi, A. Yli-Urpo, and P. K. Vallittu, "Shear bond strength of Bis-GMA resin and methacrylated dendrimer resins on silanized titanium substrate," Dental Materials, vol. 21, no. 3, pp. 287-296, 2005.

[16] F. Beari, M. Brand, P. Jenker et al., "Untersuchung zur zeitlichen Strukturänderung organofunktioneller Silane in verdünnten wässrigen Lösungen," Journal of Organometallic Chemistry, vol. 625, p. 208, 2001.

[17] M. N. Aboushelib, H. Mirmohamadi, J. P. Matinlinna, E. Kukk, H. F. Ounsif, and Z. Salameh, "Innovations in bonding to zirconia-based materials. Part II: focusing on chemical interactions," Dental Materials, vol. 25, no. 8, pp. 989-993, 2009.

[18] W. Kollenberg, Technische Keramik, Volkan, Essen, Germany, 2004.

[19] K.-J. M. Söderholm and S.-W. Shang, "Molecular orientation of siliane at the surface of colloidal silica," Journal of Dental Research, vol. 72, no. 6, pp. 1050-1054, 1993.

[20] I. D. Sideridou and M. M. Karabela, "Effect of the amount of 3-methacyloxypropyltrimethoxysilane coupling agent on physical properties of dental resin nanocomposites," Dental Materials, vol. 25, no. 11, pp. 1315-1324, 2009. 

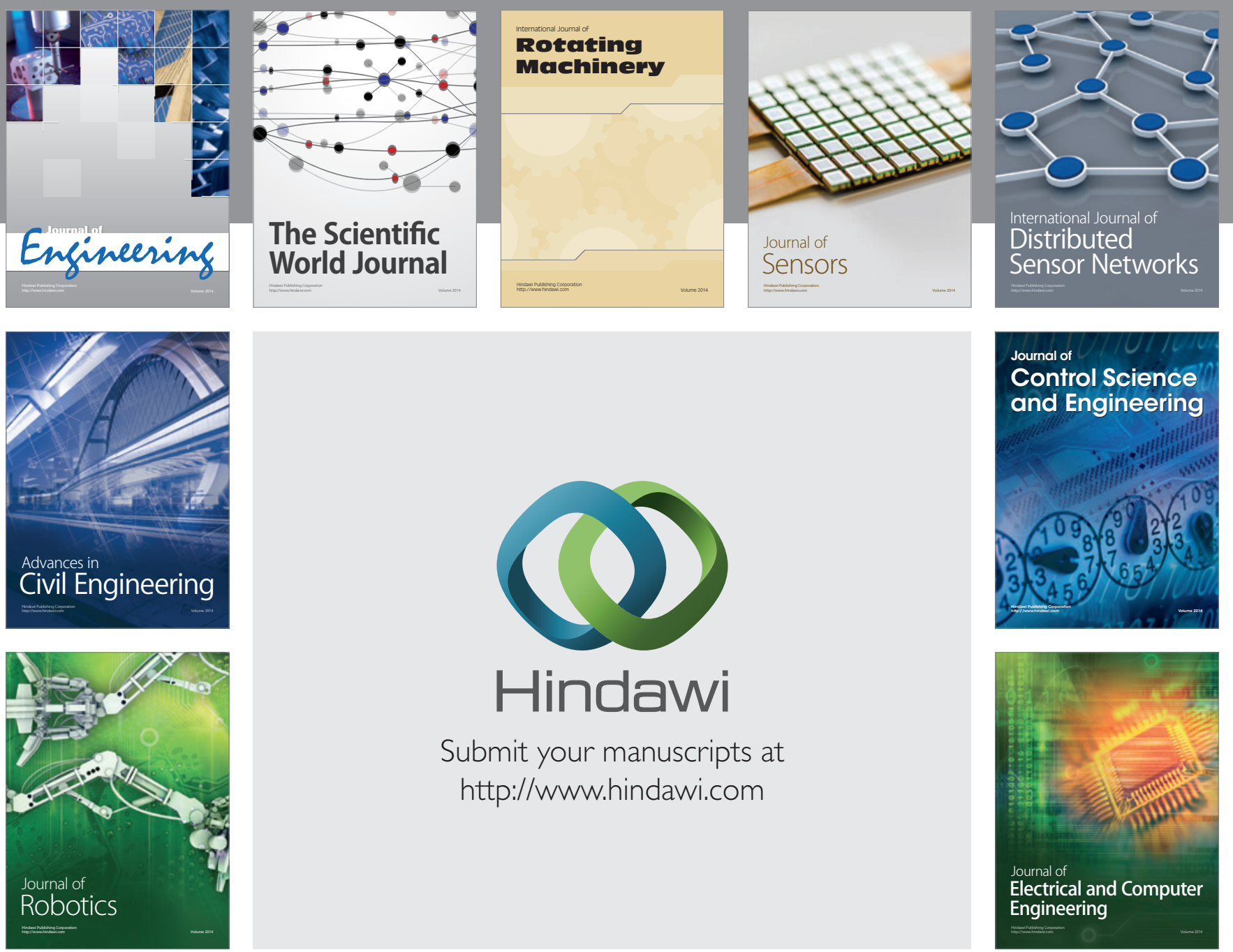

Submit your manuscripts at

http://www.hindawi.com
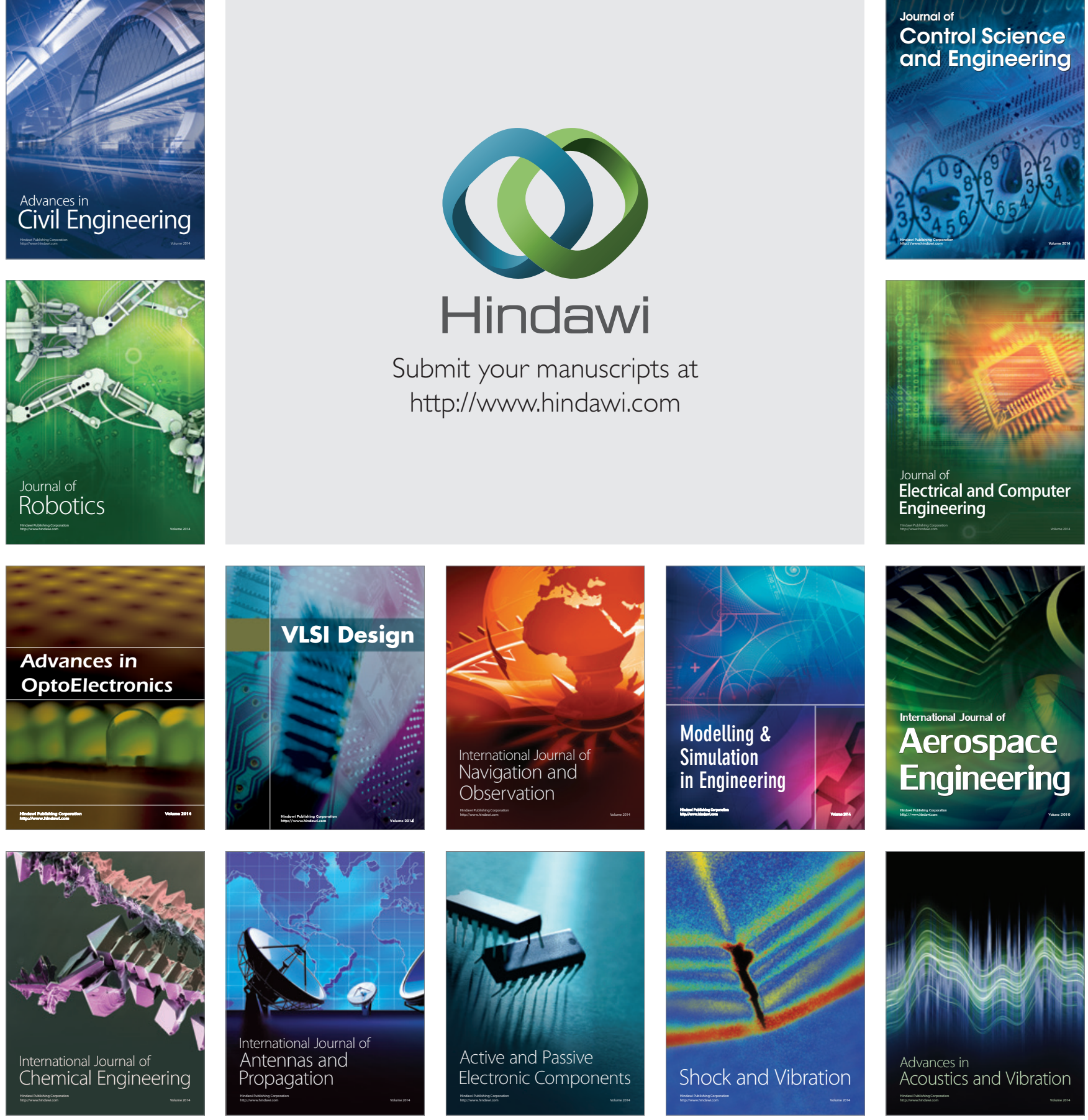